\title{
The Small Magellanic Cloud in the far infrared ${ }^{\star}$
}

\section{Global properties}

\author{
K. Wilke ${ }^{1}$, U. Klaas ${ }^{1}$, D. Lemke ${ }^{1}$, K. Mattila ${ }^{2}$, M. Stickel ${ }^{1}$, and M. Haas ${ }^{1}$ \\ 1 Max-Planck-Institut für Astronomie, Königstuhl 17, 69117 Heidelberg, Germany \\ ${ }^{2}$ Helsinki University Observatory, Tähtitorninmäki, PO Box 14, 0014 University of Helsinki, Finland
}

Received 24 July 2003 / Accepted 7 October 2003

\begin{abstract}
We present global IR properties of the Small Magellanic Cloud using an ISOPHOT $170 \mu \mathrm{m}$ map and re-visited IRAS data. The integrated $170 \mu \mathrm{m}$ flux is $14950 \pm 2300 \mathrm{Jy}$, and the integrated IR flux is $F_{1-1000}=7.48 \times 10^{-10} \mathrm{~W} \mathrm{~m}^{-2}$. Discrete sources contribute $28 \%, 29 \%$, and $36 \%$ to the integrated flux at $60 \mu \mathrm{m}, 100 \mu \mathrm{m}$, and $170 \mu \mathrm{m}$, respectively. Most of the total emission arises from diffuse emission regions surrounding the HII regions. The SED can be modelled by the composition of a $45 \mathrm{~K}$, a $20.5 \mathrm{~K}$ and a $10 \mathrm{~K}$ blackbody component with emissivity index $n=2$. A color temperature map is constructed from the ISOPHOT $170 \mu \mathrm{m}$ and the IRAS $100 \mu \mathrm{m}$ HiRes maps. The average dust temperature is $T_{\mathrm{D}}=20.3 \mathrm{~K}$. The total dust mass is found to be $M_{\mathrm{D}}=7.8 \times 10^{5} M_{\odot}$, yielding a gas-to-dust mass ratio $M_{\mathrm{gas}} / M_{\text {dust }} \approx 540$, a value 25 times lower than found by former studies. The global star formation rate is estimated to $S F R_{\text {total }} \approx 0.05 M_{\odot} \mathrm{yr}^{-1}$. The comparison with other irregular galaxies reveals the SMC as quiet with no peculiar FIR properties.
\end{abstract}

Key words. galaxies: Magellanic Clouds - ISM: general - ISM: dust, extinction

\section{Introduction}

In Wilke et al. (2003) (Paper I hereafter), a map of the Small Magellanic Cloud (SMC) obtained with ISOPHOT (Lemke et al. 1996) in the new $170 \mu \mathrm{m}$ wavelength band was presented. In order to treat the ISO and IRAS data in an identical and reproducible way we obtained IRAS high resolution maps at 12, 25, 60 and $100 \mu \mathrm{m}$ by requesting HiRes processing at IPAC. The similar spatial resolution and the application of an algorithm, which was capable of automated, repeatable photometry, even for irregular sources, allowed the examination of statistical aspects of the source distribution in the five IR bands.

This second paper focuses on global aspects and properties of the dust in the SMC, in particular the search for colder dust which may have been missed in the analysis of the IRAS data. In a forthcoming Paper III we will present and discuss maps of the dust-to-gas ratio and the link to the metallicity, as well as maps of $\tau_{170} / A_{\mathrm{V}}$.

In Sect. 2 we present a compilation of our IRAS and ISOPHOT integrated fluxes and other IR and submm photometry yielding a total SED. We discuss the superposition of

Send offprint requests to: U. Klaas, e-mail: klaas@mpia.de

* Based on observations with ISO, an ESA project with instruments funded by ESA Member States (especially the PI countries: France, Germany, The Netherlands and the UK) and with the participation of ISAS and NASA. several components with different temperatures and their relative contribution to the total luminosity and dust mass.

In Sect. 3 we compute the FIR star formation rate (SFR) and compare it with results obtained from observations at other wavelengths, especially $\mathrm{H}_{\alpha}$ observations.

Section 4 deals with the results obtained from a $170 / 100 \mu \mathrm{m}$ color temperature map which is based on the $170 \mu \mathrm{m}$ ISOPHOT map and the $100 \mu \mathrm{m}$ IRAS HiRes data.

Since the SMC is the best-studied dwarf irregular galaxy, due to its proximity, Sect. 5 compares the SMC with other irregular galaxies observed with ISO. In addition, comparisons are made with the Milky Way. These comparisons mainly address the questions of star formation rates (SFR), dust temperatures $T_{\mathrm{D}}$ and masses $M_{\mathrm{D}}$, total gas content, gas-to-dust ratios, and metallicities.

\section{Global parameters}

\subsection{Integrated FIR flux densities}

In Table 1 we present a compilation of the MIR, FIR, submm and $\mathrm{mm}$ integrated flux densities currently available from photometry with ISO, IRAS, $\mathrm{COBE}^{1}$, and the TopHat balloon-borne telescope (Silverberg et al. 2003;

\footnotetext{
1 All COBE data sets are developed by the NASA Goddard Space Flight Center under the guidance of the COBE Science Working Group and were provided by the NSSDC.
} 
Table 1. Integral MIR, FIR and submm photometry of the SMC. References: SEA00 = Stanimirovic et al. (2000); SCH88 = Schwering (1988); REA88 = Rice et al. (1988); AEA03 = Aguirre et al. (2003). IR fluxes are color corrected (cc) according to a power law fit to the referring spectral points (see text for more details), except for REA88 where the color corrections were calculated using a twotemperature thermal model fit to the measured SED. Note that the integral ISO $170 \mu \mathrm{m}$ flux density is computed after correcting for the incompleteness of the ISO map (indicated by the "**), as it is explained in the footnote to Table 3. The contribution of the $\mathrm{CO}$ emission line at $1300 \mu \mathrm{m}$ to the TopHat band is estimated to be $0.6 \%$.

\begin{tabular}{|c|c|c|c|}
\hline$(\mu \mathrm{m})$ & $\begin{array}{c}\text { flux density } \\
\pm \text { uncertainty } \\
\text { (Jy) }\end{array}$ & $\begin{array}{c}\text { cc fluxes } \\
\pm \text { uncertainty } \\
(\mathrm{Jy})\end{array}$ & $\begin{array}{l}\text { instrument, } \\
\text { reference }\end{array}$ \\
\hline 12 & & $80 \pm 30$ & DIRBE, SEA00 \\
\hline 12 & & $105 \pm 30$ & HIRAS, SEA00 \\
\hline 12 & & $147 \pm 26$ & IRAS, SCH88 \\
\hline 12 & $67 \pm 15 \%$ & $83 \pm 15 \%$ & IRAS, REA88 \\
\hline 12 & $130 \pm 40$ & $140 \pm 44$ & IRAS, this study \\
\hline 25 & & $460 \pm 18$ & DIRBE, SEA00 \\
\hline 25 & & $310 \pm 170$ & HIRAS, SEA00 \\
\hline 25 & & $385 \pm 56$ & IRAS, SCH88 \\
\hline 25 & $270 \pm 15 \%$ & $256 \pm 15 \%$ & IRAS, REA88 \\
\hline 25 & $370 \pm 50$ & $400 \pm 55$ & IRAS, this study \\
\hline 60 & & $8450 \pm 370$ & DIRBE, SEA00 \\
\hline 60 & & $6700 \pm 1060$ & HIRAS, SEA00 \\
\hline 60 & & $7170 \pm 957$ & IRAS, SCH88 \\
\hline 60 & $6689 \pm 15 \%$ & $7338 \pm 15 \%$ & IRAS, REA88 \\
\hline 60 & $7450 \pm 250$ & $7490 \pm 250$ & IRAS, this study \\
\hline 100 & & $15800 \pm 2720$ & DIRBE, SEA00 \\
\hline 100 & $16480 \pm 2222$ & & DIRBE, AEA03 \\
\hline 100 & & $13600 \pm 1930$ & HIRAS, SEA00 \\
\hline 100 & & $13900 \pm 1810$ & IRAS, SCH88 \\
\hline 100 & $15022 \pm 15 \%$ & $15506 \pm 15 \%$ & IRAS, REA88 \\
\hline 100 & $12700 \pm 600$ & $12650 \pm 600$ & IRAS, this study \\
\hline 140 & & $14000 \pm 5600$ & DIRBE, SEA00 \\
\hline 140 & $20030 \pm 920$ & & DIRBE, AEA03 \\
\hline 170 & $14950^{*} \pm 2300^{*}$ & & $\begin{array}{l}\text { ISOPHOT, } \\
\text { this study }\end{array}$ \\
\hline 240 & & $9600 \pm 4400$ & DIRBE, SEA00 \\
\hline 240 & $12070 \pm 540$ & & DIRBE, AEA03 \\
\hline 476 & $3200 \pm 810$ & & TopHat, AEA03 \\
\hline 652 & $1620 \pm 290$ & & TopHat, AEA03 \\
\hline 750 & $950 \pm 190$ & & TopHat, AEA03 \\
\hline 1224 & $320 \pm 80$ & & TopHat, AEA03 \\
\hline
\end{tabular}

Aguirre et al. 2003). Some data sets were reduced with several alternative techniques.

Our flux values are obtained by integrating over the whole area of the SMC down to a certain flux limit by summing up all relevant map pixels. It is crucial to define a conservative lower threshold. If it is too low, a strong increase of flux will be caused by adding up too much noise. This is in particular true for the short wavelength IRAS maps where the HiRes algorithm produces a stripy and fluctuating background. We achieved the definition of the surface brightness limits firstly by lowering the threshold in small steps $\left(0.1 \mathrm{MJy} \mathrm{sr}^{-1}\right)$ and in this process tracking the increase of the total flux, and secondly by inspecting the maps with the different lower intensity cuts until the noise became clearly visible. These lower limits differ from band to band. Due to the faintness and patchiness of the extended emission and the subtraction of the strong Zodiacal Light contribution at 12 and $25 \mu \mathrm{m}$, they are higher for these two bands $\left(1.7 \mathrm{MJy} \mathrm{sr}^{-1}\right)$ than for the long wavelength bands ( $1.5 \mathrm{MJy} \mathrm{sr}^{-1}$ at $60 \mu \mathrm{m}, 1.1 \mathrm{MJy} \mathrm{sr}^{-1}$ at 100 and $\left.170 \mu \mathrm{m}\right)$. The uncertainties were determined from the variation of the fluxes derived from the thresholds around the finally consolidated values. They should therefore provide a reliable estimate of the range which may be covered by the integrated SMC flux density.

The color correction factors for the re-visited IRAS data are taken from the IRAS Explanatory Supplement (Beichmann 1985). Since among the data sets from former studies the HIRAS results by Stanimirovic et al. (2000) are based on the most advanced data reduction process, we tried to perform identical color corrections to make our numbers directly comparable. Stanimirovic (1999) used color correction factors of $0.93,0.92,0.99$, and 1.0 for the $12 \mu \mathrm{m}, 25 \mu \mathrm{m}, 60 \mu \mathrm{m}$, and $100 \mu \mathrm{m}$ IRAS bands, respectively, which were obtained by fitting a power-law to the observed integrated SED. Exponents $\alpha=-2.0 \ldots-2.5$ yielded satisfying fits. For our IRAS results factors of $0.92,0.92,0.995$, and 1.005 have to be applied to the four IRAS bands.

The DIRBE fluxes are from COBE/DIRBE maps with a resolution of $\approx 0.7^{\circ}$, see Stanimirovic et al. (2000) for a detailed description of this dataset. The HIRAS fluxes are taken from the work of Stanimirovic et al. (2000) and are based on a combination of several IRAS survey scans with 14 Pointed (i.e. non-survey) Observations (POs). These data were reduced using the Pyramid Maximum Entropy (PME) routine of the HIRAS software (Bontekoe et al. 1994) to reconstruct images with a resolution approaching the diffraction limit of IRAS $(\approx 1$ arcmin). Fluxes were also obtained from the non-survey IRAS POs without the HIRAS method (Schwering 1988). Finally, IRAS fluxes published in the catalog by Rice et al. (1988) were derived using co-added IRAS maps. Recently Aguirre et al. (2003) presented reanalyzed DIRBE photometry and new photometry in the 4 bands at $245 \mathrm{GHz}(1224 \mu \mathrm{m})$, $400 \mathrm{GHz}(750 \mu \mathrm{m}), 460 \mathrm{GHz}(652 \mu \mathrm{m})$, and $630 \mathrm{GHz}(476 \mu \mathrm{m})$ obtained with the TopHat balloon-borne telescope with a beam of $20^{\prime}$.

The fluxes derived by our study are in good agreement with the results from other IRAS and COBE/DIRBE studies, and the $170 \mu \mathrm{m}$ point provides an accurate constraint in the maximum of the SED.

The separation of the relative contributions from diffuse and discrete sources to the integrated emission is difficult due to the growth procedure in the HIIphot program (see Paper I for a detailed description) which allows for the inclusion of some 

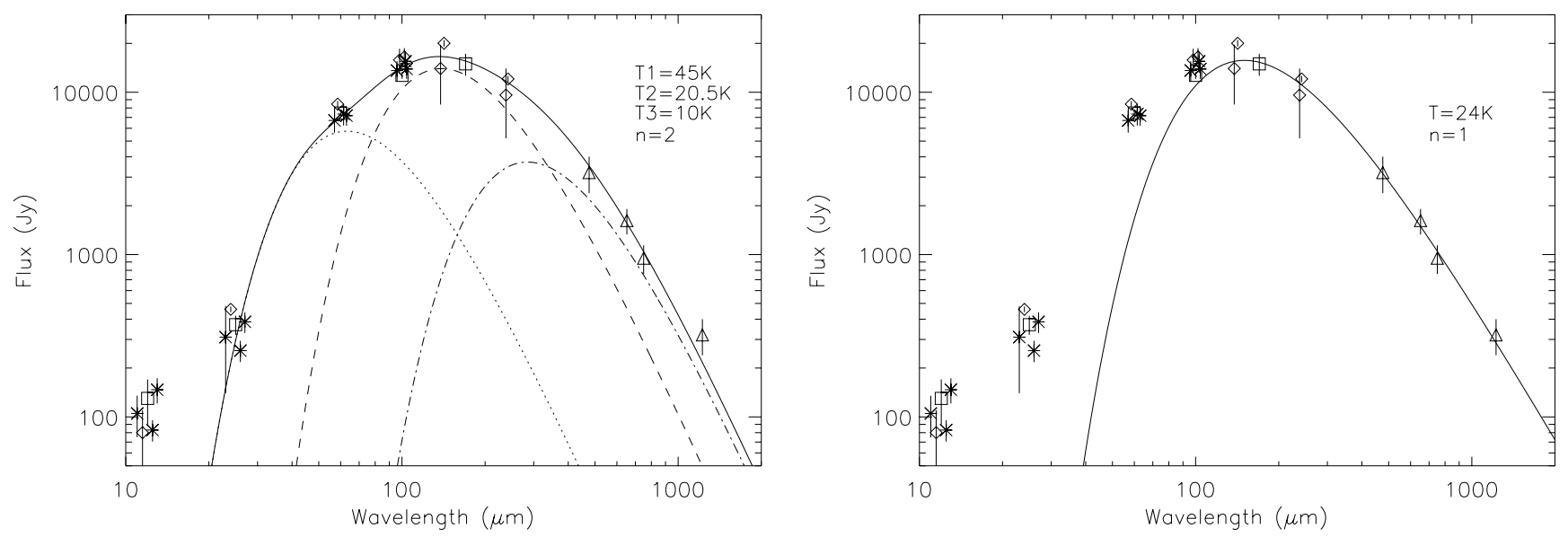

Fig. 1. 10 to $1000 \mu \mathrm{m}$ SED of the SMC established from the integrated fluxes compiled in Table 1. Squares: our photometry derived from IRAS HiRes maps and the ISOPHOT $170 \mu \mathrm{m}$ map; asterisks: IRAS photometry from previous studies; diamonds: COBE/DIRBE photometry; triangles: TopHat photometry. In case of multiple photometry at a certain wavelength the data points have been slightly shifted in wavelength against each other for better visibility. Uncertainties of each photometric point are indicated. The left panel shows the modeling of the SED by three modified blackbodies (emissivity exponent $n=2$ ) with the temperatures $45,20.5$, and $10 \mathrm{~K}$, respectively. The right plot shows the fit of the long wavelength part $(\lambda \geq 140 \mu \mathrm{m})$ with a single modified blackbody with an emissivity exponent $n=1$ and $T=24 \mathrm{~K}$.

extended emission around HII regions as well. Applying the flux limits down to which we allow the potential sources to allocate pixels, we found that "discrete" sources contribute $28 \%$, $29 \%$, and $36 \%$ to the integrated flux densities at $60 \mu \mathrm{m}, 100 \mu \mathrm{m}$, and $170 \mu \mathrm{m}$, respectively. Since at $12 \mu \mathrm{m}$ and $25 \mu \mathrm{m}$ the integrated flux is mainly originating from stellar and point-like sources, the contribution of the diffuse component is uncertain. For $12 \mu \mathrm{m}$ and $25 \mu \mathrm{m}$, we estimate the contribution from the compact sources to lie in the range 15-35\%.

In Fig. 1 we display the 10 to $1000 \mu \mathrm{m}$ SED of the SMC. Aguirre et al. (2003) found that the best fit of their submm data is achieved with an emissivity index $n=0.91 \pm 0.15$ and a dust temperature $T_{\mathrm{D}}=29.5 \pm 2.7 \mathrm{~K}$. We confirm that $n \approx 1$ gives a smooth fit to the 140 to $1200 \mu \mathrm{m}$ data points (Fig. 1 right). Our analysis suggests, however, a lower dust temperature $T_{\mathrm{D}} \approx 24 \mathrm{~K}$.

How physically realistic is the adoption of an emissivity index $n=1$ and a one temperature component fit for the FIR and submm SEDs of galaxies? Does it mean that the grain size distribution in the SMC is peculiar and different from that in other galaxies, since quite a number of investigations come to the conclusion that an index $n \approx 2$ is more applicable (e.g. Lagache et al. 1998 for our Galaxy, Bianchi et al. 1999 for normal galaxies, Chini et al. 1989 for Markarian galaxies and Klaas et al. 2001 for ULIRGs) and that the FIR and submm SEDs are better described by at least two temperature component models (e.g. Dunne \& Eales 2001; Klaas et al. 2001). Dupac et al. (2003) found from PRONAOS measurements that $n$ is varying with $T_{\mathrm{d}}$, having values $1.6 . .2 .4$ for cold $(11-20 \mathrm{~K})$ and $0.8 \ldots 1.6$ for warm $(35 \ldots 80 \mathrm{~K})$ regions.

When $n=2$ is adopted, it is obvious that the submm part can only be fitted if a very cold dust component of $T_{\mathrm{d}} \approx 10 \mathrm{~K}$ is added. In Fig. 1 left we show the fit of the SMC SED by the superposition of three modified blackbodies $\left(T_{\mathrm{D}}=45\right.$, $20.5,10 \mathrm{~K}$ ) including such a very cold component. With regard to the motivation to adopt $20.5 \mathrm{~K}$ for the temperature of the second component we refer to Sect. 4 . The hottest component of $T_{\mathrm{d}} \approx 45 \mathrm{~K}$ provides a good envelope to the 25 and $60 \mu \mathrm{m}$ data points. This would correspond to the maximum 100/170 color temperatures found for the brightest HII regions. However, it has to be noted that in this regime the emission comes mainly from transiently heated Very Small Grains (VSGs), which can only be approximately modelled by a blackbody since they are not in thermal equilibrium (cf. Li \& Draine 2002).

In Fig. 2 we demonstrate the SED model, which results when the range of temperatures over the SMC $100 \mu \mathrm{m} / 170 \mu \mathrm{m}$ color temperature map, $T_{\mathrm{D}} \approx 14 \ldots 43 \mathrm{~K}$, is taken into account (see Sect. 4). The emission by each map pixel $i$ is weighted with its optical depth $\tau_{170, i}$. The total flux is computed as

$$
F_{\text {total }}(\lambda)=\Omega \cdot \sum_{i} \tau_{170, i}\left(\frac{170}{\lambda}\right)^{n} B\left(\lambda, T_{D, i}\right), n=2
$$

with $\Omega$ being the solid angle of a map pixel (30") and $\tau_{170}$ being determined for each pixel $i$ as

$\tau_{170, i}=\frac{I_{170, i}}{B_{170, i}\left(T_{\mathrm{D}, 100 / 170, i}\right)}$.

The SED outlined by the dashed line in the top panel of Fig. 2 is only slightly broader than the $20.5 \mathrm{~K}$ component dominating the three component model in Fig. 1 left panel. Both arise from emission of large grains. The $100 \mu \mathrm{m} / 170 \mu \mathrm{m}$ color temperature SED model can not describe the short wavelength part where its contribution is $10 \%$ at $60 \mu \mathrm{m}$ and only $1 \%$ at $20 \mu \mathrm{m}$. The emission shortward of $80 \mu \mathrm{m}$ is dominated by VSGs.

\subsection{Total FIR flux densities and luminosities}

Applying the formula of Helou et al. (1988) to our re-visited IRAS data at $60 \mu \mathrm{m}$ and $100 \mu \mathrm{m}$ yields

$$
\begin{aligned}
F_{40-120} & =1.26 \times 10^{-14} \times\left[2.58 F_{60}+F_{100}\right] \mathrm{W} \mathrm{m}^{-2} \\
& =4.02 \times 10^{-10} \mathrm{~W} \mathrm{~m}^{-2}
\end{aligned}
$$



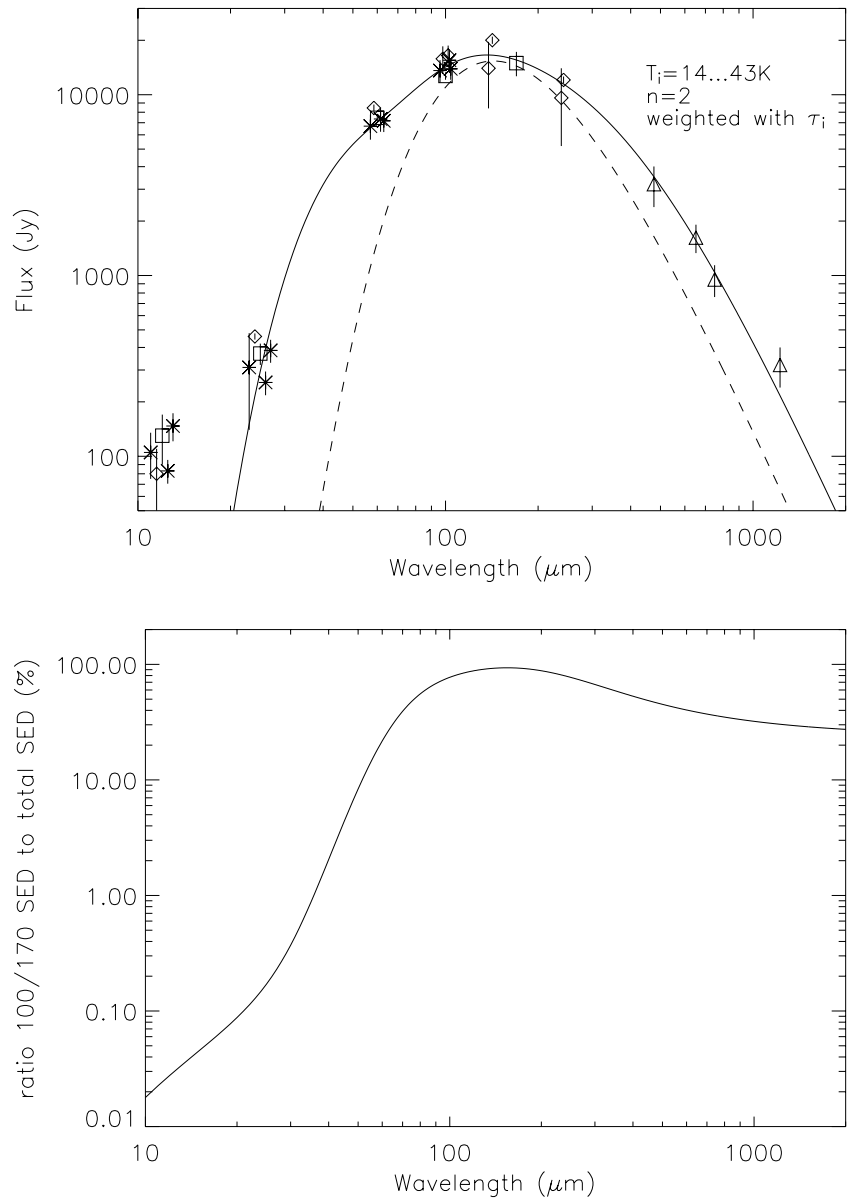

Fig. 2. Contribution of the emission of big grains in the temperature range $14 \ldots 43 \mathrm{~K}$ to the total $10-1000 \mu \mathrm{m}$ SED of the SMC. Upper panel: the SED of the big grain emission (dashed line) was computed as the sum of the emission from each map pixel taking into account the variation of the optical depth $\tau_{\mathrm{i}}$ and the color temperature $T_{\mathrm{i}}$ (see text for more details). The photometric data points and the total SED envelope (solid line) of the three component model from Fig. 1 left panel are displayed, too. The lower panel shows the wavelength dependent percentage of the big grain emission relative to the total FIR emission.

The additional ISO $170 \mu \mathrm{m}$ measurement enables us to determine the integrated infrared flux $F_{40-220}$, according to Stickel et al. (2000):

$$
\begin{aligned}
F_{40-220}= & 1.34 \times 10^{-14} \\
& \times\left[2.58 F_{60}+1.00 F_{100}+0.63 F_{170}\right] \mathrm{W} \mathrm{m}^{-2} \\
= & 5.54 \times 10^{-10} \mathrm{~W} \mathrm{~m}^{-2} .
\end{aligned}
$$

Compared with $F_{40-120}$, adding the cold FIR component at $170 \mu \mathrm{m}$ contributes $\approx 37 \%$ to the total FIR flux in the range from $40 \mu \mathrm{m}$ to $220 \mu \mathrm{m}$.

The total integrated IR flux $F_{1-1000}$ can be written as (Stickel et al. 2000)

$F_{1-1000}=1.35 F_{40-220} \mathrm{~W} \mathrm{~m}^{-2}=7.48 \times 10^{-10} \mathrm{~W} \mathrm{~m}^{-2}$.

This expression refers to the emission of big grains in the temperature range $20 \ldots 80 \mathrm{~K}$ and therefore does not account for the $12 \mu \mathrm{m}$ and $25 \mu \mathrm{m}$ contributions coming from Very Small Grains and PAH emission.
The total luminosity is then given by

$L_{1-1000}=4 \pi D^{2} \cdot F_{1-1000}=3.21 \times 10^{34} \mathrm{~W}=8.4 \times 10^{7} L_{\odot}$

where $D=60 \mathrm{kpc}$ is the luminosity distance of the SMC.

The contributions of the three modified blackbody functions presented in the previous section and Fig. 1 are: $L_{1-1000}\left(T_{1}=45 \mathrm{~K}\right)=3.5 \times 10^{7} L_{\odot}$

$L_{1-1000}\left(T_{2}=20.5 \mathrm{~K}\right)=3.9 \times 10^{7} L_{\odot}$

$L_{1-1000}\left(T_{3}=10 \mathrm{~K}\right)=0.5 \times 10^{7} L_{\odot}$

which yields a total of

$L_{1-1000}=7.9 \times 10^{7} L_{\odot}$.

The $45 \mathrm{~K}$ component makes up $44 \%$ of the total FIR luminosity, the $20.5 \mathrm{~K}$ component $49 \%$ and the $10 \mathrm{~K}$ component only $7 \%$.

\subsection{Total dust mass}

The SED analysis for $n=2$ showed that the dust emission cannot be described by one single sharply defined temperature which is also confirmed below by the range of temperatures found in the $100 \mu \mathrm{m} / 170 \mu \mathrm{m}$ color map. Formulae of the dust mass calculation which make use of the FIR luminosity have a high dependence on the mean dust temperature $\left(\propto T^{-6}\right.$ in the case of $n=2$ ). A more reliable method is therefore to derive the dust mass for each component individually via the classical formula

$M_{\mathrm{D}}=\frac{1}{\kappa_{v}(n)} \cdot \frac{D^{2} \cdot F_{v}}{B_{v}(T)}$

Since we have a complete (model) SED for each component, we can calculate the dust masses for $1300 \mu \mathrm{m}$, making use of the opacity $\kappa_{1300}(n=2)=0.37 \mathrm{~cm}^{2} \mathrm{~g}^{-1}$ (Klaas et al. 2001):

$T=45 \mathrm{~K}, \quad F_{1300}=0.82 \mathrm{Jy} \Rightarrow M_{\mathrm{D}}=5.9 \times 10^{2} M_{\odot}$

$T=20.5 \mathrm{~K}, F_{1300}=39.6 \mathrm{Jy} \Rightarrow M_{\mathrm{D}}=7.3 \times 10^{4} M_{\odot}$

$T=10 \mathrm{~K}, F_{1300}=135.5 \mathrm{Jy} \Rightarrow M_{\mathrm{D}}=7.1 \times 10^{5} M_{\odot}$.

This gives a total dust mass

$M_{\mathrm{D}, \mathrm{SMC}}=7.8 \times 10^{5} M_{\odot}$.

Here the $45 \mathrm{~K}$ component makes up only $0.08 \%$ of the total dust mass, the $20.5 \mathrm{~K}$ component $9 \%$ and the $10 \mathrm{~K}$ component $91 \%$ !

Another possibility is to make use of the pixel-wise information of the $100 \mu \mathrm{m} / 170 \mu \mathrm{m}$ color temperature map presented in Sect. 4 and calculate:

$M_{\mathrm{D}}=\frac{D^{2}}{\kappa_{v}(n)} \cdot \sum_{i} \frac{S_{v, i}}{B_{v}\left(T_{170 / 100, i}\right)}$

With $\kappa_{170}(n=2)=21.625 \mathrm{~cm}^{2} \mathrm{~g}^{-1}$ and restricting the color temperature calculation to reliable surface brightness values of $I_{100} \geq 6 \mathrm{MJy} \mathrm{sr}^{-1}$ and $I_{170} \geq 4 \mathrm{MJy} \mathrm{sr}^{-1}$, this yields

$M_{\mathrm{D}, \mathrm{SMC}} \geq 1.76 \times 10^{5} M_{\odot}$.

Since we have seen in the SED analysis that the 100 and $170 \mu \mathrm{m}$ emission cannot account for a significant amount 
of the submm emission, the total dust mass is certainly considerably higher. Therefore, we adopt $M_{\mathrm{D}}=7.8 \times 10^{5} M_{\odot}$ as our currently best estimate. With a total gas mass of $4.2 \times 10^{8} M_{\odot}$ (Stanimirovic 1999), we obtain an average gas-to-dust-ratio of

$M_{\text {gas }} / M_{\text {dust }}=540$

from the model with $n=2$, which we consider as the more applicable one, as discussed above.

The earlier studies based on IRAS $60 \mu \mathrm{m} / 100 \mu \mathrm{m}$ color temperatures and without the contribution of the cold and very cold dust components yielded dust masses of 1.4...1.8 $\times$ $10^{4} M_{\odot}$ and gas-to-dust ratios of $1.1 \ldots 1.6 \times 10^{4}$ (Schwering 1988; Lisenfeld \& Ferrara 1998; Stanimirovic et al. 2000). Our new value for the gas-to-dust ratio is therefore a factor of $\approx 25$ smaller than the previous ones. The single component model with $n=1, T=24 \mathrm{~K}$ would yield $\left(\kappa_{1300}(n=1)=\right.$ $\left.1.9 \mathrm{~cm}^{2} \mathrm{~g}^{-1}\right)$ :

$T=24 \mathrm{~K}, \quad F_{1300}=241.8 \mathrm{Jy} \Rightarrow M_{\mathrm{D}}=7.1 \times 10^{4} M_{\odot}$,

a dust mass value only a factor 4 larger than the previous ones.

Compared with the values of our Galaxy, where Dobroski et al. (1994) found $140 \pm 50$ for the inner Galaxy, $190 \pm 60$ for the outer Galaxy, and $160 \pm 60$ as an average value, the gas-todust ratio of the SMC is 3.5 times larger. We remark that the range of the gas-to-dust ratio we find now is more consistent with the metallicity of the SMC, which is about 10 times lower than that of the Milky Way. We will follow up this issue in more detail in our forthcoming Paper III where we will also consider the spatial variations of the gas-to-dust ratio.

\section{Star formation rates}

\subsection{FIR SFRs}

Star formation takes place in dense clouds of molecular gas and dust. In the extreme case nearly the total bolometric luminosity is re-radiated in the FIR. Only light from stars formed near the edges of the clouds can escape directly and is responsible for the emission in the optical and UV range.

Then, according to Kennicutt (1998), the global star formation rate (SFR) is related to the FIR luminosity $L_{\mathrm{FIR}}$ via

$$
\frac{S F R_{\mathrm{FIR}}}{1 M_{\odot} \mathrm{yr}^{-1}}=\frac{L_{\mathrm{FIR}}}{5.8 \times 10^{9} L_{\odot}} \text {. }
$$

This expression is based on the starburst synthesis models of Leitherer \& Heckmann (1995) and holds for the assumption of solar abundances, the Salpeter IMF, and the adoption of the mean luminosities for continuous starbursts which last $10 \mathrm{Myr} . .100 \mathrm{Myr}$. The formula implies that the bolometric luminosity is dominated by the young stars and that the cold dust re-radiates all of it in the FIR. Though not all conditions are fulfilled for the SMC, the Kennicutt (1998) formula gives

$S F R_{\mathrm{SMC}, \mathrm{FIR}}=0.015 M_{\odot} \mathrm{yr}^{-1}$.

Recent studies by Popescu et al. (2000) suggest the percentage of light re-radiated in the FIR to be $\approx 30 \%$ for late-type diskdominated galaxies and only $\approx 15 \%$ for earlier types. Due to small opacities in most normal galaxies, some stellar radiation is able to escape the star forming region directly and will not be re-radiated by dust in the FIR. As a consequence, the intrinsic SFRs will be underestimated when considering the FIR luminosity only. Taking into account this effect means that the conversion factor would be three times higher, i.e.

$$
S F R_{\mathrm{SMC}, \text { total }} \approx 0.05 M_{\odot} \mathrm{yr}^{-1} \text {. }
$$

It should be stated that these factors will strongly vary with environmental and opacity effects, i.e. one cannot expect this number to be constant even in a single morphological subclass.

Part of the FIR radiation can originate from dust heated by non-ionizing stars and is not associated with recent star formation. Israel et al. (1996) have estimated that in NGC 6822, a Local Group dwarf irregular galaxy of the Magellanic type, only slightly more than $50 \%$ of the total FIR luminosity originates from dust associated with stars formed during the last 10 Myr. Almost half of the FIR emission comes from dust heated by a considerably older stellar population. In this case the above formula overestimates the SFR traced by the FIR.

Another calibration of $S F R_{\mathrm{FIR}}$ is given by RowanRobinson (1997) which makes use of the so-called dusty starburst model by Rowan-Robinson \& Efstathiou (1993). It includes a treatment of the Very Small Grains and PAHs and has proven to give an excellent fit to starburst galaxies like M 82 and NGC 6090, as well as for elliptical galaxies. This already suggests that the model can serve only as a first-order approximation to the SMC case. The model fit is adjusted to MIR measurements at $6.7 \mu \mathrm{m}$ and $15 \mu \mathrm{m}$ (ISOCAM). It yields:

$$
\begin{aligned}
& S F R_{\mathrm{SB}}=4.5 \times 10^{-10} L_{\mathrm{FIR}} M_{\odot} \mathrm{yr}^{-1} L_{\odot}^{-1} \Rightarrow \\
& S F R_{\mathrm{SMC}}=0.038 M_{\odot} \mathrm{yr}^{-1} .
\end{aligned}
$$

\subsection{SFRs from $H_{\alpha}$ emission}

The star formation rate of the SMC has been subject to several studies focusing on the $\mathrm{H}_{\alpha}$-luminosity. Kennicutt \& Hodge (1986) derive $\mathrm{H}_{\alpha}$ star formation rates according to the method given in Kennicutt (1983) - not corrected for intrinsic extinction - of

$S F R_{\mathrm{SMC}, \mathrm{H}_{\alpha}, \text { uncorrected }}=0.038 M_{\odot} \mathrm{yr}^{-1}$.

To correct for the extinction, Kennicutt \& Hodge assumed an average value of $A_{\mathrm{V}}=1.0 \mathrm{mag}$ for the HII regions. This $A_{\mathrm{V}}$ is based on comparisons between radio and Balmer line fluxes of the brightest HII regions, as given in Israel \& Kennicutt (1980). As a result, the SFR becomes considerably larger:

$$
S F R_{\mathrm{SMC}, \mathrm{H}_{\alpha}}=0.08 M_{\odot} \mathrm{yr}^{-1} \text {. }
$$

According to the authors, this is probably a conservative upper limit. For these results, the integrated $\mathrm{H}_{\alpha}$ flux was obtained by summing up the flux contributions from all discrete HII regions, correcting it for contributions from unresolved objects, and adding $15 \%$ for the contribution of extended filamentary emission.

More recent studies by Kennicutt et al. (1995) use the slightly modified conversion factors between $\mathrm{H}_{\alpha}$ luminosity 
and SFR given in Kennicutt (1994), and assume an extinction of $A_{\mathrm{V}} \approx 0.3$ mag as found by Caplan et al. (1994). This is an average value which probably overestimates the extinction in the diffuse gas, but underestimates the extinction of the brightest HII regions. As a result, the SFR for the SMC is computed to

$S F R_{\mathrm{SMC}, \mathrm{H}_{\alpha}}=0.046 M_{\odot} \mathrm{yr}^{-1}$.

\subsection{Total SFRs}

Taking the values of $S F R_{\mathrm{FIR}} \approx 0.015 M_{\odot} \mathrm{yr}^{-1}$, uncorrected for the incomplete dust absorption in the FIR, and $S F R_{\mathrm{H}_{\alpha}} \approx$ $0.038 M_{\odot} \mathrm{yr}^{-1}$, uncorrected for the dust extinction, results in an optical output $\approx 2.5$ times higher, as indicated by the studies by Popescu et al. (2000). The total star formation rate

$$
\begin{aligned}
S F R_{\mathrm{SMC}, \text { total }} & =S F R_{\mathrm{SMC}, \mathrm{FIR}}+S F R_{\mathrm{SMC}, \mathrm{H}_{\alpha}} \\
& \approx 0.053 M_{\odot} \mathrm{yr}^{-1}
\end{aligned}
$$

is in reasonable agreement with the results by Kennicutt et al. (1995). However, our SFR suggests a slightly larger average $A_{\mathrm{V}}$ than used in their study. From their photometric survey of the SMC Zaritsky et al. (2002) found an average $A_{\mathrm{V}}=$ $0.46 \mathrm{mag}$ for the population of hot stars $\left(12000 \mathrm{~K} \leq T_{\text {eff }} \leq\right.$ $45000 \mathrm{~K})$. Should only $50 \%$ of the FIR luminosity be powered by young stars, as suggested by the study of Israel et al. (1996) for NGC 6822, then

$$
\begin{aligned}
S F R_{\mathrm{SMC}, \text { total }} & =0.5 \cdot S F R_{\mathrm{SMC}, \mathrm{FIR}}+S F R_{\mathrm{SMC}, \mathrm{H}_{\alpha}} \\
& \approx 0.046 M_{\odot} \mathrm{yr}^{-1}
\end{aligned}
$$

in agreement with the results by Kennicutt et al. (1995).

\section{The $170 \mu \mathrm{m} / 100 \mu \mathrm{m}$ temperature map}

\subsection{Construction}

To generate a color temperature map the $170 \mu \mathrm{m}$ maps were created with a pixel size of $30^{\prime \prime}$ in order to allow a direct comparison with the original HiRes $100 \mu \mathrm{m}$ maps with a pixel size of $15^{\prime \prime}$. The latter ones were rebinned to $30^{\prime \prime}$ pixel size using the $2 \times 2$ block averaging routine within the IRAF environment, as explained in more detail in Paper I.

The dust color temperature $T_{\mathrm{D}}$ was calculated according to the following procedure: The ratio of the color corrected flux densities ( $K_{\mathrm{cc}}$ denote the color correction factors) of the two bands equals the ratio of the modified Planck function values for $T_{\mathrm{D}}$ :

$$
\frac{F_{v_{1}}\left(v F_{v}=\text { const. }\right) / K_{\mathrm{cc}}\left(\lambda_{1}\right)}{F_{v_{2}}\left(v F_{v}=\text { const. }\right) / K_{\mathrm{cc}}\left(\lambda_{2}\right)}=\frac{\lambda_{2}^{3+n}}{\lambda_{1}^{3+n}} \cdot \frac{\mathrm{e}^{\frac{h c}{k k_{2} T_{\mathrm{D}}}}-1}{\mathrm{e}^{\frac{h c}{k \lambda_{1} T_{\mathrm{D}}}}-1}
$$

With $\alpha_{1}=\frac{\lambda_{2}^{3+n} \cdot F_{v_{2}} / K_{\mathrm{cc}}\left(\lambda_{2}\right)}{\lambda_{1}^{3+n} \cdot F_{v_{1}} / K_{\mathrm{cc}}\left(\lambda_{1}\right)}, \beta=h c / k \lambda_{2}$, and $\gamma=h c / k \lambda_{1}$, this can be written in form of a difference:

Diff $=\alpha_{1} \cdot \mathrm{e}^{\frac{\beta}{T_{\mathrm{d}}}}-\mathrm{e}^{\frac{\gamma}{T_{\mathrm{d}}}}+\left(1-\alpha_{1}\right)$.

The exact $T_{\mathrm{d}}$ is derived by finding the root of Diff, which is done by numerical iteration.
Table 2. Uncertainties in the $T_{\mathrm{D}}$ calculation. They are calculated for the two temperatures $T_{\mathrm{D}}=20 \mathrm{~K}\left(f_{100}=1 \mathrm{Jy}, f_{170}=1.47 \mathrm{Jy}\right.$, not color corrected $)$ and $T_{\mathrm{D}}=30 \mathrm{~K}\left(f_{100}=1 \mathrm{Jy}, f_{170}=0.625 \mathrm{Jy}\right.$, not color corrected). The indicated uncertainties are maximum values which are derived by adding the relative flux uncertainty to the one flux density and subtracting it from the other one and vice versa.

\begin{tabular}{ccccc}
\hline \hline \multicolumn{4}{c}{$\Delta T$ for } \\
& $\Delta f= \pm 2 \%$ & $\Delta f= \pm 5 \%$ & $\Delta f= \pm 10 \%$ & $\Delta f= \pm 20 \%$ \\
\hline$T=20 \mathrm{~K}$ & $+0.30 \mathrm{~K}$ & $+0.82 \mathrm{~K}$ & $+1.71 \mathrm{~K}$ & $+3.77 \mathrm{~K}$ \\
& $-0.30 \mathrm{~K}$ & $-0.75 \mathrm{~K}$ & $-1.45 \mathrm{~K}$ & $-2.74 \mathrm{~K}$ \\
$T=30 \mathrm{~K}$ & $+0.72 \mathrm{~K}$ & $+1.90 \mathrm{~K}$ & $+4.09 \mathrm{~K}$ & $+9.76 \mathrm{~K}$ \\
& $-0.72 \mathrm{~K}$ & $-1.68 \mathrm{~K}$ & $-3.21 \mathrm{~K}$ & $-5.81 \mathrm{~K}$ \\
\hline
\end{tabular}

The accuracy of the derived color temperatures depends on the accuracy of the $100 \mu \mathrm{m}$ and $170 \mu \mathrm{m}$ photometry and the absolute level of the temperature. For the temperature maps relative uncertainties may depend on drift effects and flat-field accuracies. In Table 2 we show uncertainties for different temperatures depending on the assumed photometric uncertainties. The uncertainties for the major part of the map are in the range $1 \ldots 2 \mathrm{~K}$.

\subsection{Temperature regimes}

In Fig. 3 the $170 \mu \mathrm{m} / 100 \mu \mathrm{m}$ color temperature map is displayed. In the upper panel the superposition of the $170 \mu \mathrm{m}$ surface brightness contours on the $100 \mu \mathrm{m}$ surface brightness map is shown in order to demonstrate the quality of the registering. For a few bright compact sources there appears to be a small shift between the two maps. This may be due to the asymmetric beam profiles of the IRAS maps, so that the resulting color temperature gradients close to these compact sources may be artefacts. Furthermore, there are some minor artefacts at the border of the two original 2 deg $\times 2$ deg maps from which the map was assembled (see Paper I for an overview of the split of the SMC area).

The color temperature map presented in the lower panel is remarkably homogeneous, there is not much difference in $T_{\mathrm{D}}$ between the bright and faint regions. The temperature range covered is $14 \ldots 43 \mathrm{~K}$. The mean value of the total map is $T_{\mathrm{D}}=20.3 \mathrm{~K}$ with a standard deviation of $2.4 \mathrm{~K}$, the median value is $20.4 \mathrm{~K}$. The main body (the bar) of the SMC is slightly warmer than the regions of the eastern wing. For the main body the mean temperature is $20.9 \pm 1.9 \mathrm{~K}$ (median $=20.8 \mathrm{~K}$ ) for the eastern wing the mean temperature is $18.1 \pm 2.8 \mathrm{~K}$ (median $=$ $17.5 \mathrm{~K}$ ). This may be a consequence of the weaker interstellar radiation field in the wing.

In the main body the temperatures of the bright star formation regions in the south west (except the one hot spot) and in the north west are lower by a few degrees than the surroundings. This may be a consequence of the presence of molecular clouds with relatively cold dust which lowers the average dust temperatures in these regions. We will follow up this issue in the detailed spatial study of the forthcoming Paper III. 

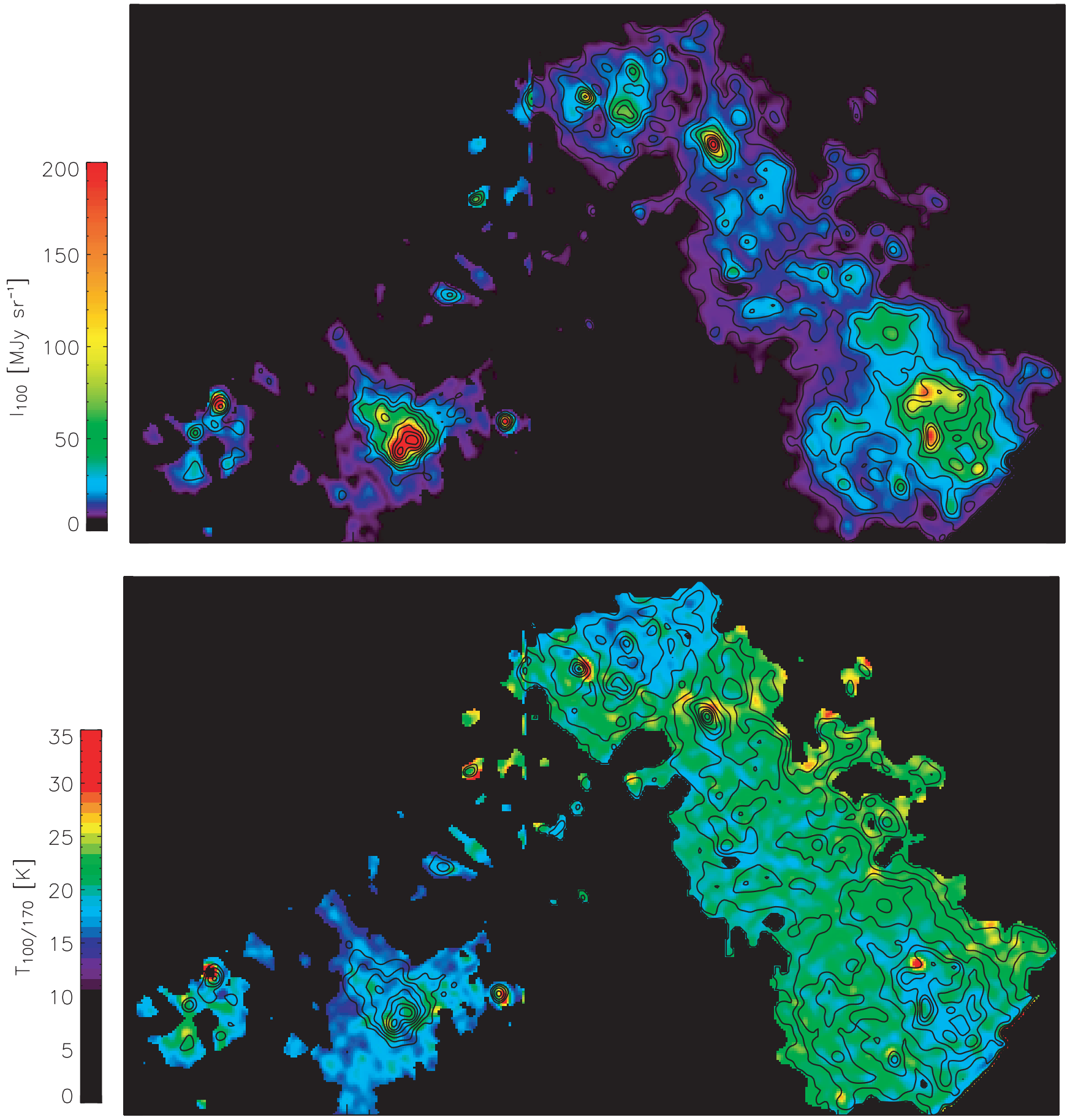

Fig. 3. $170 \mu \mathrm{m} / 100 \mu \mathrm{m}$ color temperature map of the SMC. Upper panel: $100 \mu \mathrm{m}$ surface brightness map $I_{100}$ (color) with the $I_{170}$ map overlaid in contours. Lower panel: dust color temperature map; only map pixels with $I_{100} \geq 6 \mathrm{MJy} \mathrm{sr}^{-1}$ and $I_{170} \geq 4 \mathrm{MJy} \mathrm{sr}^{-1}$ are considered in order to ensure high quality ratios; again the $170 \mu \mathrm{m}$ intensity contours are overlaid.

\subsection{Comparison with the Milky Way}

The dust temperatures of the SMC can be compared with the results of Sodroski et al. (1994) for the Milky Way. For an integrated FIR luminosity of the Galaxy of $1.1 \times 10^{10} L_{\odot}$, they find that $\approx 60 \%-75 \%$ of this emission emerges from the dust component associated with diffuse HI clouds $\left(T_{\mathrm{D}}=17 \mathrm{~K} \ldots 22 \mathrm{~K}\right)$, another $\approx 15 \%-30 \%$ arises from $T_{\mathrm{D}} \approx 19 \mathrm{~K}$ dust residing in molecular clouds, the remaining contibution $(<10 \%)$ comes from extended low-density (ELR) HII regions of temperatures up to $\approx 29 \mathrm{~K}$. Observational values for the dust temperature in the Milky Way given by Sodroski et al. (1994) are $T_{\mathrm{D}}=$ $20 \pm 1 \mathrm{~K}$ for the inner Galaxy $\left(270^{\circ}<l<350^{\circ}\right), T_{\mathrm{D}}=17 \pm 1 \mathrm{~K}$ for the outer Galaxy $\left(90^{\circ}<l<270^{\circ}\right)$, and $T_{\mathrm{D}}=19 \pm 1 \mathrm{~K}$ as an average value, based on COBE $140 \mu \mathrm{m}$ and $240 \mu \mathrm{m}$ data. For the high latitude $(|b|>10 \mathrm{deg})$ cirrus emission Lagache et al. (1998) found an equilibrium dust temperature of about $17.5 \mathrm{~K}$ with only small variations over the entire high latitude sky. 
Table 3. Comparison of global properties of the irregular galaxies SMC, IC 4662, NGC 1569, and NGC 3077.

\begin{tabular}{|c|c|c|c|c|}
\hline & SMC & IC 4662 & NGC 1569 & NGC 3077 \\
\hline Hubble type & $\mathrm{S}(\mathrm{B}) \mathrm{sm}$ pec & $\mathrm{IBm}$ & $\mathrm{Sm} \mathrm{IV/IBm}$ & I0 pec \\
\hline$A$ & $6^{\circ} \times 8^{\circ(a)}$ & $2.8^{\prime} \times 1.6^{(b)}$ & $1.44^{\prime(c)}$ & $5.4^{\prime} \times 4.5^{\prime(d)}$ \\
\hline$d$ & $60 \mathrm{kpc}^{(a)}$ & $2.1 \mathrm{Mpc}^{(b)}$ & $2.2 \mathrm{Mpc}^{(c)}$ & $3.9 \mathrm{Mpc}^{(d)}$ \\
\hline$A_{d_{\mathrm{SMC}}}$ & $6^{\circ} \times 8^{\circ}$ & $1.6^{\circ} \times 0.9^{\circ}$ & $0.9^{\circ}$ & $5.9^{\circ} \times 4.9^{\circ}$ \\
\hline$\left[M_{\odot}\right]$ & $\approx 2 \times 10^{9(a)}$ & $\approx 7.6 \times 10^{8(e)}$ & $3.3 \times 10^{8(d)}$ & $1.8 \times 10^{10(f)}$ \\
\hline$\left[M_{\odot}\right]$ & $\approx 4.2 \times 10^{8(g)}$ & $1.5 \times 10^{8(h)}$ & $1.1 \times 10^{8(d)}$ & $1.8 \times 10^{9}$ \\
\hline$\left[M_{\odot}\right]$ & $2 \times 10^{6(i)}$ & $1.0 \times 10^{6(h)}$ & $2 \times 10^{7(d)}$ & $8 \times 10^{5(k)}$ \\
\hline$\left[M_{\odot}\right]$ & $7.8 \times 10^{5(l)}$ & $1.5 \times 10^{5(m)}$ & $1.6 \ldots 7.8 \times 10^{5(n)}$ & $4.5 \times 10^{5(m)}$ \\
\hline$M_{\mathrm{gas}} / M_{\mathrm{dust}}$ & $\approx 540$ & $\approx 1000$ & $\approx 440$ & $\approx 2400$ \\
\hline$F_{\mathrm{ISO}} @ 170 \mu \mathrm{m}$ & $14950^{*}$ & 10.5 & $28.0^{(o)}$ & 25.4 \\
\hline$F_{\text {IRAS }} @ 12,25 \mu \mathrm{m}[\mathrm{Jy}]$ & $130 / 370$ & $0.30 / 1.27$ & $0.79 / 7.09$ & $0.73 / 2.03$ \\
\hline$F_{\text {IRAS }} @ 60,100 \mu \mathrm{m}[\mathrm{Jy}]$ & $7450 / 12700$ & $8.81 / 11.90$ & $45.4 / 47.3$ & $13.65 / 26.90$ \\
\hline$L_{1-1000}$ & $8.4 \times 10^{7}$ & $1.5 \times 10^{8}$ & $4.9 \times 10^{8}$ & $1.0 \times 10^{9}$ \\
\hline$[\mathrm{K}]$ & 20.5 & 25.0 & 40.0 & 24.0 \\
\hline
\end{tabular}

The rows are defined as follows:

A:

$d:$

$A_{d, \mathrm{SMC}}$ :

$M_{\text {total }}$ :

$M_{\text {gas }}$ :

$M_{\mathrm{H}_{2}}$ :

$M_{\text {dust }}$ :

$M_{\text {gas }} / M_{\text {dust }}$ :

$F_{\text {ISO }}$ :

$F_{\text {IRAS }}$ :

$L_{1-1000}$ :

$T_{\mathrm{D}}$ :

*:

Area covered in the sky, taken from:

${ }^{(a)}$ : Westerlund (1997), ${ }^{(b)}$ : Dale et al. (2000), ${ }^{(d)}$ : NASA/IPAC Extragalactic Database (NED), in the case of NGC 1569, the Holmberg radius is taken from ${ }^{(c)}$ : Israel (1988) and references therein.

Distance.

Area covered in the sky assuming that the objects were located at the SMC distance (60 kpc).

Total mass of the object including all gas and stellar components, taken from:

${ }^{(e)}$ : Reif et al. (1982), ${ }^{(f)}$ : Thomasson \& Donner (1993).

Gaseous mass, including molecular, atomic, and ionized hydrogen, taken from:

${ }^{(g)}$ : Stanimirovic et al. (1999), ${ }^{(h)}$ : Heydari et al. (1990, model based value).

Molecular hydrogen mass, taken from:

${ }^{(i)}$ : Tumlinson et al. (2002), ${ }^{(j)}$ : Sanders et al. (1991), ${ }^{\left({ }^{k}\right)}$ : Walter et al. (2002).

Dust mass, including all available FIR contributions, taken from:

${ }^{(l)}$ : Wilke et al. (2003), ${ }^{(m)}$ : Stickel et al. (2000), ${ }^{(n)}$ : Galliano et al. (2003).

Dust-to-gas mass ratio.

ISO fluxes at $170 \mu \mathrm{m}$ taken from the serendipity survey analysis by Stickel et al. (2000) or ${ }^{(o)}$ : Hunter et al. (1989).

IRAS fluxes obtained at $12 / 25 / 60 / 100 \mu \mathrm{m}$.

Integrated IR luminosity.

Temperature of the coldest dust component.

The integrated ISO fluxes were empirically corrected for the contribution of the missing SW part of the $\mathrm{SMC}(\approx 5 \%$, i.e., $\approx 790 \mathrm{Jy}$ ) which remained unobserved during the ISO mission.

A comparison of the SMC and the Galaxy dust temperatures is not straight forward since the two systems are very different in size and structure. However, the average temperature of the SMC dust is higher indicating a more intense (harder) interstellar radiation field.

\section{Comparison with other irregular galaxies}

In dwarf irregular galaxies, the process of star formation takes place in short-lived strong bursts rather than in a continuous process. This stochastic process explains their irregular morphology to a large extent, as well as the fact that most of the galaxies of this type are chemically less evolved, i.e. have lower metallicities.

It is useful to compare our results with those of other irregular galaxies observed by ISO. Stickel et al. (2000) published a list of 115 galaxies detected at $170 \mu \mathrm{m}$ by the ISOPHOT serendipity survey (Bogun et al. 1996). According to the morphological classification taken from the NED, two sources are irregular galaxies: IC 4662, and NGC 3077. Another irregular galaxy, NGC 1569, has been examined by pointed observations and is a source of the serendipity survey data base (Galliano et al. 2003).

Table 3 compares some global and infrared properties of these four objects. However, as their morphological subtypes indicate, the objects are different with regard to their overall structure and appearance.

IC 4662 is the object most similar to the SMC since it is classified as barred. This galaxy is an interesting case as it does not belong to any known group. IC 4662 has already been subject to extensive studies, especially regarding its star formation 
rate (Heydari-Malayeri et al. 1990; Hunter et al. 2001; Dale et al. 2000).

NGC 1569 is a nearby irregular dwarf galaxy and a member of the low-galactic latitude IC 342/Maffei 1/Maffei 2/Dw 1 group which contains more than 15 galaxies. This galaxy is at present in the aftermath of a massive starburst, indicated by the morphology of its body which mainly consists of single starforming knots. The FIR emission of this object is comparably strong for a dwarf galaxy, the continuum spectrum indicates that the dust in this galaxy is exposed to intense radiation fields (Lisenfeld et al. 2002).

NGC 3077 belongs to an interacting quartet of galaxies, the other members of which are NGC 2976, M 82, and M 81. This ensemble, the M 81 group, is embedded in a large cloud of atomic hydrogen, with bridges of gas connecting M 81 to M 82 and NGC 3077 . Indeed, $M_{\text {gas }}$ of NGC 3077 is relatively high. This morphological scenario is apparently similar to the case of the so-called Magellanic Stream (Thomasson \& Donner 1993).

Since the other three irregular galaxies are located at much greater distances than the SMC, we scale their apparent areas in the sky to the distance of the SMC. When projected to the SMC distance, NGC 3077 approximately covers the same area as the SMC, while IC 4662 and NGC 1569 remain considerably smaller. On the other hand, the total masses of the SMC, NGC 1569, and IC 4662 are comparable, while the mass of NGC 3077 is estimated roughly ten times larger (Thomasson \& Donner 1993).

The gas-to-dust mass ratios for the SMC and NGC 1569 are a factor of 2 to 5 smaller than for IC 4662 and NGC 3077. This may be simply a consequence of the fact that for the former two objects dust masses have been estimated including submm data, while for the latter two the dust masses where estimated utilizing the luminosity and the average $100 \mu \mathrm{m} / 170 \mu \mathrm{m}$ color temperatures only. As discussed in the analysis of the SMC data this can have a significant impact on the resulting dust masses. The dust masses for IC 4662 and NGC 3077 must therefore be considered as lower limits.

It is remarkable that the two smaller galaxies - NGC 1569 and IC 4662 - have a $100 / 60 \mu \mathrm{m}$ flux ratio of $\approx 1$, while the other two, the SMC and NGC 3077, show values of $\approx 2$. This suggests that the former two objects are in a more active (i.e. star-forming) state. This holds especially for NGC 1569 where an average temperature of $\approx 40 \mathrm{~K}$ is found when fitting a double blackbody component to the SED. It is the only object with a $60 \mu \mathrm{m}$ flux as high as the $100 \mu \mathrm{m}$ one, hinting at the exceptional state of activity this galaxy is undergoing. Compared with the other three irregular galaxies, the SMC appears to be the least active object.

\section{Conclusions}

Integral fluxes for the SMC were derived from the ISOPHOT $170 \mu \mathrm{m}$ map and the reprocessed IRAS HiRes maps. With complementary data from COBE/DIRBE and the TopHat submm/mm instrument the 10 to $1200 \mu \mathrm{m}$ SED was established and the total luminosity in this wavelength range derived. A model with three temperature components was fitted to the SED leading to higher dust masses and a smaller gas-to-dust mass ratio. The total SFR was determined taking into account dust extinction and incomplete dust absorption. A $100 \mu \mathrm{m} / 170 \mu \mathrm{m}$ color temperature map traces the temperature distribution of the big grains. Finally, the SMC IR properties were compared with those of other irregular galaxies. Quantitatively, the results are the following:

1. The FIR fluxes derived from our analysis complement those from earlier studies.

2. With $F_{1-1000}=7.48 \times 10^{-10} \mathrm{~W} \mathrm{~m}^{-2}, L_{1-1000}=8.4 \times 10^{7} L_{\odot}$.

3. Discrete sources contribute $28 \%, 29 \%$, and $36 \%$ to the integrated flux at $60 \mu \mathrm{m}, 100 \mu \mathrm{m}$, and $170 \mu \mathrm{m}$, respectively, most of the total emission arises from diffuse emission regions surrounding the HII regions.

4. The SED established from MIR, FIR, and submm/mm photometry can be modelled between 25 and $1200 \mu \mathrm{m}$ by three modified $(n=2)$ blackbodies with temperatures of 45 , 20.5 , and $10 \mathrm{~K}$, respectively. A single temperature $(T=$ $24 \mathrm{~K}$ ) model with an emissivity index $n=1$ fits only the $140-1200 \mu \mathrm{m}$ part of the SED well.

5. The three component dust model allows the derivation of more reliable dust masses. The existence of the cold and very cold dust components, not seen in earlier studies based on IRAS photometry, increases the dust mass to $M_{\mathrm{D}, \mathrm{SMC}}=7.8 \times 10^{5} M_{\odot}$. The big grains in the temperature range $14 \ldots 43 \mathrm{~K}$ make up $\approx 1.8 \times 10^{5} M_{\odot}$.

6. The new gas-to-dust mass ratio of the SMC is 540, this is 25 times less than derived in previous studies.

7. The best estimate for the total SFR of the SMC is $S F R_{\text {total }} \approx$ $0.05 M_{\odot} \mathrm{yr}^{-1}$. Only $\leq 30 \%$ of the bolometric luminosity of young stars is absorbed by dust and re-radiated in the FIR.

8. The mean $100 \mu \mathrm{m} / 170 \mu \mathrm{m}$ color temperature of the big dust grains is $20.3 \mathrm{~K}$ which is higher than for the Milky Way indicating a harder ISRF. There is a gradient in dust temperature from the bar $(20.9 \mathrm{~K})$ to the eastern wing $(18.1 \mathrm{~K})$.

9. Compared to the other irregular galaxies IC 4662, NGC 1569, and NGC 3077, the SMC appears to be the least active one.

Acknowledgements. This work was performed at the ISOPHOT Data Centre at MPIA which is supported by Deutsches Zentrum für Luftund Raumfahrt e.V. (DLR) with funds of Bundesministerium für Bildung und Forschung, grant No. 50QI0201, and by the Max-PlanckGesellschaft, supporting the scientific data reduction. We thank the referee for constructive comments. This research has made use of the Simbad Database, operated at CDS, Strasbourg, France, and NASA's Astrophysics Data System Abstract Service. We used the NASA/IPAC Extragalactic Database (NED) which is operated by the Jet Propulsion Laboratory, California Institute of Technology, under contract with the National Aeronautics and Space Administration.

\section{References}

Aguirre, J. E., Bezaire, J. J., Cheng, E. S., et al. 2003, ApJ, 596, 273 Beichmann, C. A., Neugebauer, G., Habing, H. J., Clegg, P. E., \& Chester, T. J. 1985, Infrared Astronomical Satellite (IRAS) catalogs and atlases, Explanatory Supplement, Jet Propulsion Lab., California Institute of Technology, Pasadena, California

Bianchi, S., Davies, J. I., \& Alton, P. B. 1999, A\&A, 344, L1

Bogun, S., Lemke, D., Klaas, U., et al. 1996, A\&A, 315, L71 
Bontekoe, T. R., Koper, E., \& Kester, D. J. M. 1994, A\&A, 284, 1037

Caplan, J., Ye, T., Deharveng, L., Turtle, A. J., \& Kennicutt, R. C. 1996, A\&A, 307, 403

Chini, R., Krügel, E., Kreysa, E., \& Gemuend, H.-P. 1989, A\&A, 216, L5

Dale, D. A., Silbermann, N. A., Helou, G., et al. 2000, AJ, 120, 583

Dunne, L., \& Eales, S. A. 2001, MNRAS, 327, 697

Dupac, X., Bernard, J.-P., Boudet, N., et al. 2003, A\&A, 404, L11

Galliano, F., Madden, S. C., Jones, A. P., et al. 2003, A\&A, 407, 159

Helou, G., Khan, I. R., Malek, L., \& Boehmer, L. 1988, ApJS, 68, 151

Heydari-Malayeri, M., Melnick, J., \& Martin, J.-M. 1990, A\&A, 234, 99

Hunter, D. A., Throson, H. A. Jr., Casey, S., \& Harper, D. A. 1989 , ApJ, 341, 697

Hunter, D. A., Kaufman, M., Hollenbach, D. J., et al. 2001, ApJ, 553, 121

Israel, F. P., \& Kennicutt, R. C. 1980, Ap. Lett., 21, 1

Israel, F. P. 1988, A\&A, 194, 24

Israel, F. P., Bontekoe, T. R., \& Kester, D. J. M. 1996, A\&A, 308, 723

Kennicutt, R. C. 1983, ApJ, 272, 54

Kennicutt, R. C., \& Hodge, P. W. 1986, ApJ, 306, 130

Kennicutt, R. C. 1994, in Violent Star Formation from 30 Doradus to QSO's, ed. G. Tenorio-Tagle (Cambridge: Cambridge University Press)

Kennicutt, R. C., Bresolin, F., Bomans, D. J., Bothun, G. D., \& Thompson, I. B. 1995, AJ, 109, 594

Kennicutt, R. C. 1998, ApJ, 498, 541

Klaas, U., Haas, M., Müller, S. A. H., et al. 2001, A\&A, 379, 823

Lagache, G., Abergel, A., Boulanger, F., \& Puget, J.-L. 1998, A\&A, 333, 709

Leitherer, C., \& Heckmann, T. M. 1995, ApJS, 96, 9

Lemke, D., Klaas, U., Abolins, J., et al. 1996, A\&A, 315, L64

Li, A., \& Draine, B. T. 2002, ApJ, 576, 762
Lisenfeld, U., \& Ferrara, A. 1998, ApJ, 496, 145

Lisenfeld, U., Israel, F. P., Stil, J. M., \& Sievers, A. 2002, A\&A, 382, 860

Popescu, C. C., Misirotis, A., Kylafis, N. D., et al. 2000, A\&A, 362, 138

Reif, K., Mebold, U., Goss, W. M., Woerden, \& H. van Siegmann, B. 1982, A\&AS, 50, 451

Rice, W., Lonsdale, C. J., Soifer, B. T., et al. 1988, ApJS, 68, 91

Rowan-Robinson, M., \& Efstathiou, A. 1993, MNRAS, 263, 675

Rowan-Robinson, M., Mann, R. G., \& Oliver, S. J. 1997, MNRAS, 289,490

Sanders, D. B., Scoville, N. Z., \& Soifer, B. T. 1991, ApJ, 370, 158

Schwering, P. 1988, Ph.D. Thesis, Sterrewacht Leiden

Silverberg, R. F., Aguirre, J. E., Bezaire, J. J., et al. 2003, The long duration flight of the TopHat experiment, SPIE, 4857, 195

Sodroski, T. J., Bennett, C., Boggess, N., et al. 1994, ApJ, 428, 638

Stanimirovic, S. 1999, Ph.D. Thesis, University of Western Sydney Nepean

Stanimirovic, S., Staveley-Smith, L., Dickey, J. M., Sault, R. M., \& Snowden, S. L. 1999, MNRAS, 302, 417

Stanimirovic, S., Staveley-Smith, L., van der Hulst, J. M., et al. 2000, MNRAS, 315, 791

Stickel, M., Lemke, D., Klaas, U., et al. 2000, A\&A, 359, 865

Thomasson, M., \& Donner, K. J. 1993, A\&A, 272, 153

Tumlinson, J., Shull, J. M., Rachford, B. L., et al. 2002, ApJ, 566, 857

Walter, F., Weiss, A., Martin, C., \& Scoville, N. 2002, AJ, 123, 225

Walterbros, R. A. M., \& Schwering, P. B. W. 1987, A\&A, 180, 27

Westerlund, B. E. 1997, The Magellanic Clouds (Cambridge, UK: Cambridge University Press), 32

Wilke, K., Stickel, M., Haas, M., et al. 2003, A\&A, 401, 873 (Paper I)

Zaritsky, D., Harris, J., Thompson, I. B., Grebel, E. K., \& Massey, P. 2002, AJ, 123, 855 